\title{
Research Article \\ Endogenous Instability in Credit-Constrained Emerging Economies with Leontief Technology
}

\author{
Cristiana Mammana and Elisabetta Michetti \\ Department of Economic and Financial Institutions, University of Macerata, 62100 Macerata, Italy \\ Correspondence should be addressed to Elisabetta Michetti, michetti@unimc.it
}

Received 20 March 2008; Accepted 16 June 2008

Recommended by Masahiro Yabuta

This work provides a framework to analyze the role of financial development as a source of endogenous instability in emerging economies subject to moral hazard problems. We propose and study a dynamic model describing a small open economy with a tradeable good produced by internationally mobile capital and a country specific input, using Leontief technology. We demonstrate that emerging markets could be endogenously unstable since large capital inflows increase risk and exacerbate asymmetric information problems, according to empirical evidences. Using bifurcation and stability analysis, we describe the properties of the system attractors, we assess the plausibility for complex dynamics and, we find out that border collision bifurcations can emerge due to the fact that the state space is piecewise smooth. As a consequence, when a fixed or periodic point loses its stability, the final dynamics may become suddenly chaotic. This fact may explain how financial crises occurred in emerging economies.

Copyright (C) 2008 C. Mammana and E. Michetti. This is an open access article distributed under the Creative Commons Attribution License, which permits unrestricted use, distribution, and reproduction in any medium, provided the original work is properly cited.

\section{Introduction}

The facts leading to the financial crisis in the emerging markets of South-East Asia in summer 1997 have shown how a crisis can emerge after a boom in the fundamentals, therefore they open new theoretical approaches to financial crises and a need for new explanations. In the case of emerging markets, we witness a new phenomenon because, differently from past crises (like Mexico 1994 or European Monetary System 1992), such crisis was characterized by a large capital inflows with borrowing excess in a financial liberalizationcontext, a fast economic growth driven by fundamentals with poverty reduction (Asian miracle) and an increase in the financial risk assumed without a prudential regulation and a financial supervision system.(The fact that macroeconomic factors, especially a boom in lending, played a key role in the vulnerability of emerging markets to financial crises, has been discussed in World Economic Outlook [1]. Furthermore Corsetti et al. [2], state that in Thailand the boom in lending caused problems in its financial sector. In Hong Kong and 
Singapore, the development has been accompanied by strong risk supervision and control, so the financial crisis was prevented.)

According to such considerations, a model that can explain such financial crisis must prove that an inversion in the real aggregates with a fall in investment and save, is not only possible but can also appear in an unpredictable and sudden way when the economy goes through financial development.

In this work, we present a framework that provides an explanation to these peculiar events according to the balance-sheet view to crises. (Contributions to this line of research are in Aghion et al. [3-5], Caballero and Krishnamurthy [6], Cespedes et al. [7] and Krugman [8].) Using the discrete dynamic system theory, we prove that economies are endogenously unstable when going through a phase of financial development if the entrepreneurs face credit constraint due to moral hazard problems, as proved by Bernanke and Gertler [9]. (The relevance of asymmetric information problems in such crises has been largely recognized by Furman and Stiglitz [10] and Mishkin [11]. The fact that the level cash flow of the firm plays an important role in the investment is widely recognized in Hubbard [12] and in Bernanke et al. [13].)

Many authors considered that financial constraints on firms due to asymmetric information considerations can play a role in the propagation of the business cycle. For instance, in Azariadis and Smith [14] and Kiyotaki and Moore [15], the authors studied a closed economy and they showed that credit constraints can lead to oscillations. Differently, considering open economies, in Aghion et al. [4], the authors studied a credit-constrained model where firms have debt both in domestic and foreign currency, and they prove that the economy can easily suffer a financial crisis. A revised version of this monetary model is offered in a later work of the same authors, Aghion et al. [16], where they proved that the existence of nominal price rigidities can lead to multiple equilibria.

While the models in Aghion et al. [4, 16] focused on the monetary sector, we study a real economy model of the kind considered in Aghion et al. [17], in Aghion et al. [18], and in Caballé et al. [19].

In Aghion et al. [17], the authors developed a simple macroeconomic model where the combination between moral hazard problems in capital markets and unequal access to investment opportunities across individuals generates endogenous and permanent fluctuations in aggregate GDP, investment, and interest rates. In their work, the endogenous cycles are the product of two separate forces: high investment begets high profits and high investment but, at the same time, high investment pushes up interest rates and reduces future profits and investment. We will consider a similar mechanism in which high investment pushes up the price of the constant country-specific input and reduces future profits and investment.

Similarly, in Aghion et al. [18], the authors developed a dynamic, open, creditconstrained, real economic model to conclude that financial underdeveloped or very developed economies are stable while at an intermediate level of financial development, economies may be unstable. Anyway in that work the authors only provide numerical simulations showing the existence of a stable 2-period cycle. However, the existence of either cycles of higher period or chaotic dynamics is not proved from their work.

In this paper, we prove instead the existence of a noncanonical route to chaos due to border collision bifurcations. Border collision bifurcations occur in piecewise smooth maps when a fixed point collides with a borderline separating two smooth regions. The discontinuous change in the Jacobian elements results in many atypical bifurcation phenomena, like a periodic orbit turning directly into a chaotic orbit, or multiple attractors 
coming into existence or going out of existence as the parameter is varied across some critical value, and so forth. (About such kinds of noncanonical route to chaos, see Nusse and Yorke [20].)

Finally, in Caballé et al. [19], the authors studied the dynamics exhibited by an economic model based on the real side describing a small open economy subject to credit constraint due to moral hazard problems using a Cobb-Douglas production function. They proved that complex dynamics are exhibited at intermediate level of financial development to conclude that economies experiencing a process of financial development are more unstable than both very underdeveloped and very developed economies. We present a similar model to that proposed in Caballé et al. [19] while assuming Leontief technology, and consequently the macroeconomic model here studied is a piecewise-linear dynamic system.

The main results of the study herewith conducted are that economies with very developed or very undeveloped financial markets have a unique globally structurally stable, fixed point; while emerging markets could be endogenously unstable. In fact, we prove that an intermediate level of financial development does exist such that the system exhibits a border collision bifurcation that opens a two-piece chaotic region. When entering in the aperiodic region, the chaotic properties of the attractor make the evolution of the system sensitively depending on the initial condition, the dynamics are unpredictable and structurally unstable, so perturbations on the parameters (exogenous shocks) produce large and persistent effects. In the chaotic region, we observe also periodic windows so that the dynamics are predictable even though the period of the periodic orbit could be so high as to make impossible the distinction between such a cycle and a proper aperiodic orbit.

The properties we demonstrate allow us to argue that when going through a phase of financial development, the dynamics shown by the system could drastically change and pass from a stable fixed point to chaotic, aperiodic, unpredictable behavior. A similar result has been reached by Caballé et al. [19] only for economies characterized by a Cobb-Douglas technology, while we show that their results still hold also in the case of Leontief technology, that is, if there is no substitutability between production factors.

The basic mechanism we describe is a combination of two opposite forces deriving from an increase in the investment level. Firstly, a greater investment leads to greater output and profits. Higher profits improve credit worthiness and fuel borrowing thus leading to greater investment. Simultaneously, this boom increases the demand for country-specific input and rises its relative price. This rise in input prices leads to lower profits and reduces credit worthiness, borrowing and investment with a subsequent fall in aggregate output. So we will be able to conclude that financial development may destabilize economies that start from an intermediate level of financial development according to the experience documented in a number of countries. (E.g., in the years leading up to the crisis of the early 1980's in Southern Cone countries, there is evidence that profits in the tradeable sector sharply deteriorated due to a rise in domestic input prices. See Galvez and Tybout [21], Petrei and Tybout [22], and De Melo et al. [23].)

In fact, the endogenous explanation we pursue in this work is consistent with the experience of several emerging markets where the liberalization process has taken place (like South-East Asia) where, as a result of a rapid financial liberalization process, capital inflowed in large quantities allowing rapid growth in lending and a boom in investment. When large capital inflows are associated with growing imbalances, the crisis came, and most of these forces got reversed: capital flowed out, currency collapsed, real-estate prices dropped, lending stopped, and investment collapsed. (See World Bank [24] for a description of the link between capital-flow reversal and currency crises.) It is however important to 
emphasize that the aim of this paper is not to explain exactly what happened in some specific country but rather to propose and study a unified, dynamic, macroeconomic model that awards a central role to financial constraints and financial development.

The paper is organized as follows. In Section 2 we present the model. In Section 3 we study the qualitative dynamics of the model: we prove the global stability of economies with low- or high-financial development and we assess the plausibility for instability of economies at an intermediate level of financial development. In Section 4 we present numerical simulations that enforce the results we proved in Section 3. We give our conclusions in Section 5.

\section{The model}

We consider a small open economy that produces a single tradeable good using capital $K$ and a country-specific input $Z$ (like land or real estate) whose price $p$ is expressed in terms of produced goods. We assume that the supply of $Z$ is constant.

In such an economy, there are two categories of individuals: first the lenders who can lend their wealth to the entrepreneurs or invest in the international capital market given the international equilibrium interest rate $r=1+R>1$ but they cannot invest directly in the production, second the borrowers that are the entrepreneurs investing in the production or in the international capital market. The total output, in time $t$, produced in the economy is given by using the following Leontief technology which prevents any kind of substitution among the different inputs:

$$
y_{t}=\min \left\{\frac{K_{t}}{a}, Z\right\},
$$

where $1 / a>r$ is the capital productivity. (This hypothesis is necessary because otherwise the entrepreneurs have no incentive to invest in the production.) The tradeable good can be consumed or accumulated as productive capital for the production in the next period. We assume that capital fully depreciates after one period.

Asymmetric information considerations generate moral hazard so, according to the results reached by Bernanke and Gertler [9], entrepreneurs can borrow at most a proportional amount $\mu \geq 0$ of their own wealth at time $t, W_{t}$, that is $\mu W_{t}$. Let $L$ be the amount that entrepreneurs can borrow then, in time $t, L_{t} \leq \mu W_{t}$. The parameter $\mu$ represents the level of financial development reached by the economy. As a limit case, when $\mu=0$, entrepreneurs can invest only their own wealth, while the bigger $\mu$ is, as the more possibility they have to borrow from the capital market, and so the financial system is well developed. (Holmstrom and Tirole [25] also consider the direct relationship between capital market rules and the level of the financial development due to moral hazard considerations.) Since the maximum amount entrepreneurs can borrow from capital markets is fixed, the total investment $I_{t}$ in each time is upper bounded by

$$
I_{t}=(1+\mu) W_{t}
$$

At each period, entrepreneurs maximize their profits and this program determines their optimal demand $z_{t}$ of the input Z. Given the production function (2.1), the profit maximization implies that the entrepreneurs' optimal demand of the country-specific input is $z_{t}=K_{t} / a$, where

$$
K_{t}=I_{t}-p_{t} z_{t}
$$


that is, the difference between the total amount invested and the cost of the production factor demanded. equation:

In an equilibrium situation, it must be $\left(I_{t}-p_{t} z_{t}\right)=a z_{t}$ so we reach the following

$$
I_{t}-p_{t} z_{t}=a z_{t}
$$

Since $Z$ is constant, each of the following cases can be verified.

$\left(Z>K_{t} / a\right)$. There is an excess in the supply of $Z$, so its price (in terms of produced goods) is equal to zero. In this case, production is given by substituting (2.3) in (2.1), placing $p_{t}=0$, and considering that the credit constraint holds with (2.2). So we have that

$$
y_{t}=\frac{1}{a}(1+\mu) W_{t}
$$

$\left(Z \leq K_{t} / a\right)$. There is an excess in the demand of $Z$ (its price is positive) and production in bounded by $y_{t}=Z$. In case we can derive the equilibrium, price $p_{t}$ of the production factor $Z$ considering that both relation (2.4) and the credit constraint (2.2) hold and placing $z_{t}=Z$. Finally we have

$$
p_{t}=\frac{(1+\mu) W_{t}-a Z}{Z} \text {. }
$$

Relation (2.6) states a positive relationship between $p_{t}$ and $W_{t}$. It depends on the existence of the credit constraint (2.2) in the sense that greater wealth implies greater investment via credit constraint and so greater demand of $Z$ and, consequently, its price increases.

Now we can derive the dynamic model describing the economy. Considering that the price of the country-specific production factor $p_{t}$, the investment, $I_{t}$, and the production, $y_{t}$, are all expressed in terms of entrepreneurs wealth $W_{t}$, the dynamic system is the net wealth produced by entrepreneurs and saved by consumers, available for the next period, that is given by

$$
W_{t+1}=(1-\alpha)\left(e+y_{t}-r \mu W_{t}\right)
$$

where $\alpha \in(0,1)$ is the consumption rate, so $(1-\alpha)$ is the constant fraction the consumers save of their own wealth, and $e \geq 0$ is an exogenous income. This relation can be understood considering that at time $t$ entrepreneurs borrow, invest, produce, and pay their debt $r \mu W_{t}$ while consumers save.

Now we have to consider the role played by the credit constraint. To do this, we need to study three different cases.

(1) If the financial system is well developed, entrepreneurs invest in the production only up to the point in which the productive investment return is equal to the capital market return so

$$
\left(y_{t}-r \mu W_{t}\right)=r W_{t}
$$

In this case, there is no pure profit, and substituting (2.8) in the dynamic equation (2.7), we obtain the following increasing function of the entrepreneurs' wealth:

$$
W_{t+1}=(1-\alpha)\left(e+r W_{t}\right)
$$

that holds for well-developed economies. 
(2) If the financial system is underdeveloped, the investment-which is constraineddoes not absorb the total supply of the country specific factor $Z$ so its relative price is zero. Greater current wealth implies greater investment, and therefore greater production and because of $p=0$, greater profits and future wealth. In this case, the dynamic system is given by substituting (2.5) in the wealth dynamic equation (2.7) so we obtain the following increasing function:

$$
W_{t+1}=(1-\alpha)\left[e+\left(\frac{1+\mu}{a}-r \mu\right) W_{t}\right]
$$

that holds for less-developed economies.

(3) Finally, if the financial system is at an intermediate level of development, the investment absorbs the supply of the country-specific production factor $Z$ and, according to the Leontief production function, the production $y_{t}=Z$. Substituting such equation in the dynamic relation (2.7), we have the following decreasing function:

$$
W_{t+1}=(1-\alpha)\left[e+Z-r \mu W_{t}\right]
$$

that holds for intermediate financial developed economies.

Equations (2.10), (2.11), and (2.9) describe the dynamic system for low, intermediate, and high level of financial development of the economy respectively and, similarly, low, intermediate, and high level of entrepreneurs' wealth.

From the previous considerations, we derive the map $W_{t+1}=f\left(W_{t}\right)$ that is given by (2.10) for $W_{t} \in\left[0, W^{M}\right)$, by (2.11) for $W_{t} \in\left[W^{M}, W^{m}\right)$, and by (2.9) for $W_{t} \in\left[W^{m},+\infty\right)$, where the turning points $W^{M}$ and $W^{m}$ are given by

$$
\begin{aligned}
W^{M} & =\frac{Z a}{1+\mu}, \\
W^{m} & =\frac{Z}{r(1+\mu)} .
\end{aligned}
$$

The dynamic model we want to study is given by the following continuous first-order piecewise linear map, whose iterates describe the dynamics of entrepreneur wealth we investigate:

$$
f\left(W_{t}\right)= \begin{cases}f_{1}\left(W_{t}\right)=(1-\alpha)\left[e+\left(\frac{1+\mu}{a}-r \mu\right) W_{t}\right], & 0 \leq W_{t}<W^{M} \\ f_{2}\left(W_{t}\right)=(1-\alpha)\left[e+Z-r \mu W_{t}\right], & W^{M} \leq W_{t}<W^{m} \\ f_{3}\left(W_{t}\right)=(1-\alpha)\left(e+r W_{t}\right), & W^{m} \leq W_{t}\end{cases}
$$

where $\alpha \in(0,1), \mu \geq 0,1 / a>r>1, e \geq 0$, and $Z>0$ are the economically plausible definition domains of the parameters. 


\section{Qualitative dynamics}

In this section, we study the qualitative dynamics of the continuous bimodal piecewise linear map given by (2.14) when varying its parameters. In particular we consider the case of $e>0$ and $(1-\alpha) r<1$. (The study of the special case $e=0$ needs a partially different approach because the system would also have a fixed point at the origin even if it is always unstable. The hypothesis $(1-\alpha) r<1$ is economically plausible considering that $\alpha \gg 0$ while $r=1+\epsilon, \epsilon$ is sufficiently low.) It must be remembered that $f$ is increasing on $D_{1}=\left[0, W^{M}\right)$ and on $D_{3}=\left[W^{m},+\infty\right)$ while it is decreasing on $D_{2}=\left[W^{M}, W^{m}\right)$. (Note that if $\mu=0, f_{2}$ is constant on $D_{2}$.) Furthermore, its constant slopes in each of such pieces are, respectively, given by

$$
\begin{gathered}
f_{1}^{\prime}\left(W_{t}\right)=(1-\alpha)\left(\frac{1+\mu}{a}-r \mu\right), \\
f_{3}^{\prime}\left(W_{t}\right)=(1-\alpha) r \\
f_{2}^{\prime}\left(W_{t}\right)=-(1-\alpha) r \mu .
\end{gathered}
$$

The following proposition gives sufficient conditions on the parameter $\mu$ with respect to the other parameters of the model such that the fixed point lies on each of the three linear pieces of map (2.14).

Proposition 3.1. Let $f$ be given by (2.14) and let $e>0$ and $(1-\alpha) r<1$. Then:

(a) for all $\mu \in\left[0, \mu^{m}\right), f$ has a unique positive fixed point $W_{1}^{\star} \in D_{1}$;

(b) for all $\mu \in\left[\mu^{m}, \mu^{M}\right)$, $f$ has a unique positive fixed point $W_{2}^{\star} \in D_{2}$;

(c) for all $\mu \in\left[\mu^{M},+\infty\right), f$ has a unique positive fixed point $W_{3}^{\star} \in D_{3}$;

where $\mu^{m}=(Z a-(1-\alpha)(Z+e)) /(1-\alpha)(e+Z+Z a r), \mu^{M}=(Z(1-(1-\alpha) r)) /(1-\alpha) e r-1$, and $\mu^{M}>\mu^{m}>0$.

Proof. Let $g\left(W_{t}\right)=f\left(W_{t}\right)-W_{t}$.

To prove part (a) we consider that $g(0)=(1-\alpha) e>0$, while $g\left(W^{M}\right)=(\mu(1-\alpha)(e+$ $Z-r Z)+[(1-\alpha)(e+Z)-Z a]) /(1+\mu)$, where $g\left(W^{M}\right)<0$, for all $\mu<\mu^{m}$. So $f$ has at least one fixed point in $D_{1}$. The uniqueness in $D_{1}$ follows because $f$ is linear in $D_{1}$ with slope other than one because we are assuming $(1-\alpha) r<1$. In this case, the fixed point is given by

$$
W_{1}^{\star}=\frac{(1-\alpha) e}{1-(1-\alpha)[((1+\mu) / \alpha)-r \mu]} .
$$

Furthermore, it is the unique fixed point of $f$ in $\mathbb{R}_{+}$because $f$ is continuous, $f_{2}$ is a decreasing function and $f_{3}$ is an increasing function with slope less than one for the hypothesis $(1-\alpha) r$ $<1$.

To prove part (b) we first consider that if $\mu=\mu^{m}$ then $g\left(W^{M}\right)=0$ so the existence of the fixed point in $D_{2}$ is proven. Otherwise, if $\mu \in\left(\mu^{m}, \mu^{M}\right)$, then the same arguments we use to prove part (a) show that $g\left(W^{M}\right)>0$ while $g\left(W^{m}\right)=(\mu[r e(1-\alpha)]+[r(1-\alpha)(e+Z)-Z]) /(1+\mu)$, where $g\left(W^{m}\right)<0$ for all $\mu \in\left(\mu^{m}, \mu^{M}\right)$. So $f$ has at least one fixed point in $D_{2}$. The uniqueness in $D_{2}$ follows because $f$ is decreasing in $D_{2}$. In this case, the fixed point is given by

$$
W_{2}^{\star}=\frac{(1-\alpha)(e+Z)}{1+(1-\alpha) r \mu} .
$$


Furthermore, it is the unique fixed point of $f$ in $\mathbb{R}_{+}$because $f$ is continuous, $f_{1}$ is an increasing function with positive intercept for the hypothesis $e>0$, and $f_{3}$ is an increasing function with slope less than one for the hypothesis $(1-\alpha) r<1$.

To prove part (c) we first consider that if $\mu=\mu^{M}$, then $g\left(W^{m}\right)=0$ so the existence of the fixed point in $D_{3}$ is proven. Otherwise, if $\mu \in\left(\mu^{M},+\infty\right)$, then the same arguments we use to prove part (b) show that $g\left(W^{m}\right)>0$ while it does have a value of $W_{t}$, for instance, $\bar{W}=(1-\alpha) e /(1-(1-\alpha) r)$, where $\bar{W}>W^{m}$ because $\mu>\mu^{M}$, such that $g(\bar{W})<0$. So $f$ has at least one fixed point in $D_{3}$. The uniqueness in $D_{3}$ follows because $f$ is linear in $D_{3}$ with slope lesser than one for the hypothesis $(1-\alpha) r<1$. In such a case, the fixed point is given by

$$
W_{3}^{\star}=\frac{(1-\alpha) e}{1-(1-\alpha) r} .
$$

Furthermore, it is the unique fixed point of $f$ in in $\mathbb{R}_{+}$because $f$ is continuous and piecewise linear and $f_{1}$ is an increasing function with positive intercept for the hypothesis $e>0$.

Cases (a), (b), and (c) are depicted in Figure 1.

The following proposition states the global stability of economies at high- or lowfinancial development levels.

Proposition 3.2. Let $f$ be given by (2.14) and let $e>0$ and $(1-\alpha) r<1$. Then

(a) for all $\mu \in\left[0, \mu^{m}\right), W_{1}^{\star}$ is a globally stable fixed point;

(b) for all $\mu \in\left[\mu^{M},+\infty\right), W_{3}^{\star}$ is a globally stable fixed point.

Proof. To prove statement (a) we first consider that for all $\mu \in\left[0, \mu^{m}\right)$, the map $f$ has a unique fixed point $W_{1}^{\star}$ such that $W_{1}^{\star} \in D_{1}$, as proved in Proposition 3.1 part (a). Furthermore $f$ is a linear increasing function in $D_{1}$ and $f(0)>0$ (because of $e>0$ ) while $f\left(W^{M}\right)<W^{M}$, so its multiplier is given by $(3.1), f_{1}^{\prime} \in(0,1)$, for all $W_{t} \in D_{1}$. Then the fixed point $W_{1}^{\star} \in D_{1}$ is asintotically stable in $D_{1}$. Considering that this case set $D_{1}$ is globally attractive and positively invariant, then we conclude that $W_{1}^{\star}$ is globally stable.

To prove statement (b) we first consider that for all $\mu \in\left[\mu^{M},+\infty\right)$, the map $f$ has a unique fixed point $W_{3}^{\star} \in D_{3}$ as proved in Proposition 3.1, part (c). Now we have to consider two different cases. First, if $\mu \in\left(\mu^{M},+\infty\right)$, then $W_{3}^{\star} \in D_{3}^{\prime}$, where $D_{3}^{\prime}=D_{3} \backslash\left\{W^{m}\right\}$. Furthermore, its multiplier is given by $(3.2), f_{3}^{\prime} \in(0,1)$ for the hypothesis $(1-\alpha) r<1$, so the fixed point $W_{3}^{\star}$ is asintotically stable in the set $D_{3}^{\prime}$. The global stability is trivially proved. Secondly, if $\mu=\mu^{M}$, then $W_{3}^{\star}=W^{m}$ that is a no-differentiable point, so we cannot calculate its eigenvalue. However, $W^{m}$ is asintotically stable from the right in the sense that for all $W_{0} \in D_{3}^{\prime}$, the sequence of the iteratives converges to $W^{m}$. Furthermore, $D_{3}^{\prime}$ is positively invariant and globally attractive. So we conclude that $W_{3}^{\star} \in D_{3}$ is globally stable for all $\mu \in\left[\mu^{M},+\infty\right)$.

About such cases see, Figures 2 and 3.

As we proved, the dynamics exhibited by economies at high or low levels of financial development are tame: the generic orbit converging to the unique positive fixed point is definitively monotone. Furthermore, the economy is structurally stable, because of the hyperbolicity of the fixed point, so its behavior is predictable.

Now we have to consider the case of $\mu \in\left[\mu^{m}, \mu^{M}\right)$, that is, the case of economies at an intermediate level of financial development. First, we consider that in such a case the generic orbit that eventually converges to the fixed point (or to another attractor) is not monotone. In 


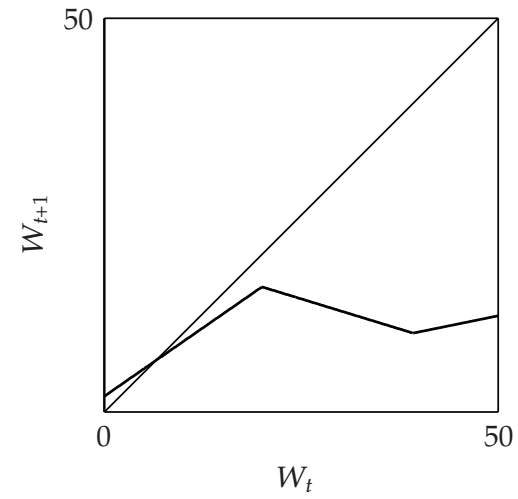

(a)

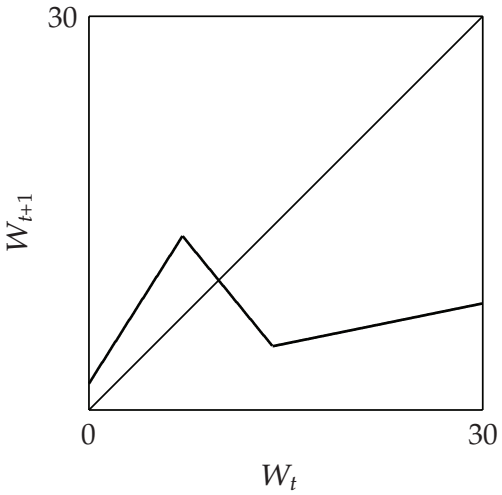

(b)

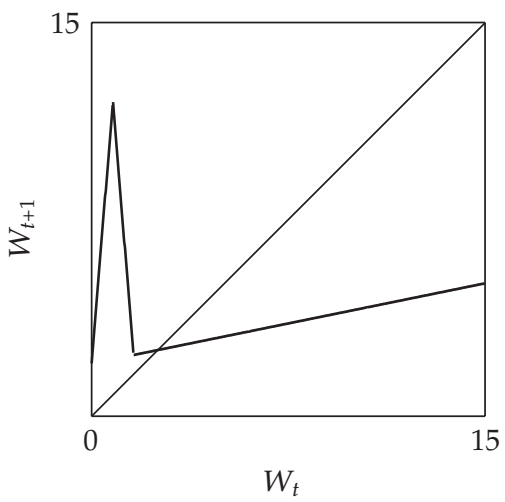

(c)

Figure 1: Scheme of $f$ as defined in (2.14) in the three cases of localization of the fixed point: in (a), $\mu=1.5$, in (b), $\mu=6$, and in (c), $\mu=60$.

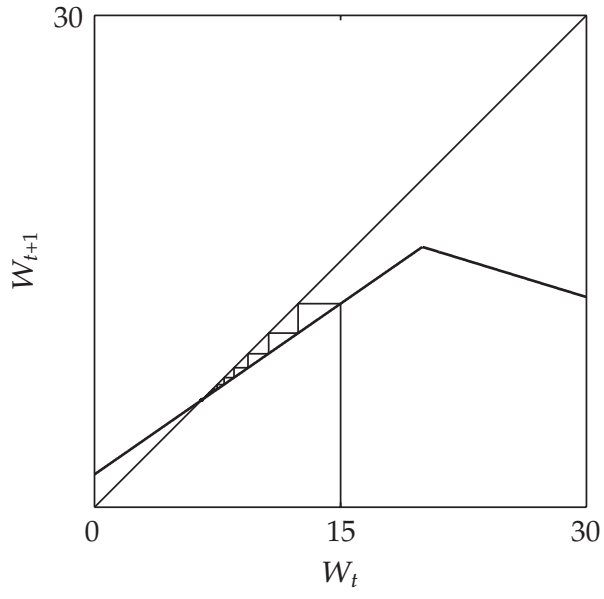

(a)

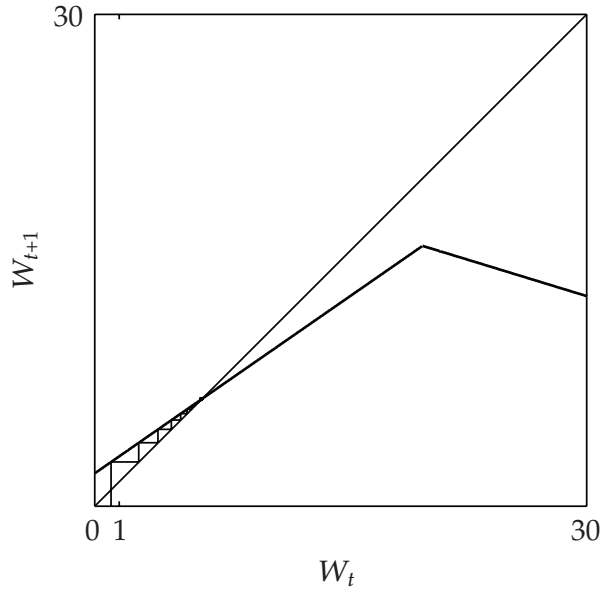

(b)

Figure 2: Koenigs Lemerary staircase diagram for two different initial conditions: in (a), $W_{0}=15 \in$ $\left(W_{1}^{\star}, W^{M}\right)$ while in (b), $W_{0}=1<W_{1}^{\star}$. In both cases $\mu=1.5$. 


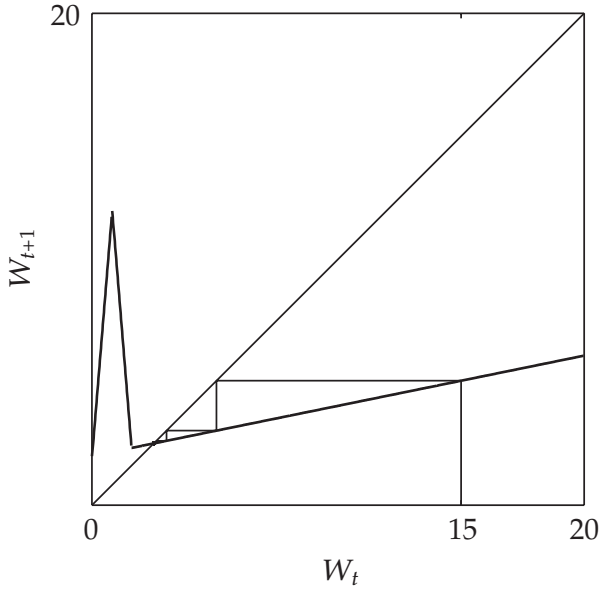

(a)

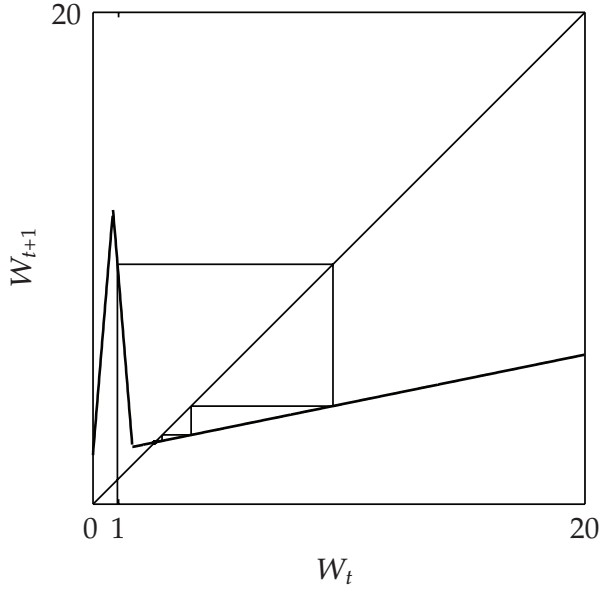

(b)

Figure 3: Koenigs Lemerary staircase diagram for two different initial conditions: in (a), $W_{0}=15>W_{3}^{\star}$ while in (b), $W_{0}=1<W_{3}^{\star}$. In both cases $\mu=60$.

fact, the only fixed point $W_{2}^{\star}$ belongs to the decreasing piece of $f$, that is, the set $D_{2}$, so every point at the right of $W_{2}^{\star}$ is mapped to its left and vice versa. So, even though the fixed point is stable, the dynamics of the trajectory is definitively oscillating and economic fluctuations are observed.

The following proposition proves the stability of economies at an intermediate level of financial development when $\mu$ is small enough (see Figure 4 panel (a)).

Proposition 3.3. Let $f$ be given by (2.14) and let $e>0$ and $(1-\alpha) r<1$. Then the fixed point $W_{2}^{\star}$ is globally stable for all $\mu \in\left[\mu^{m}, \mu^{\star}\right)$, where $\mu^{\star}=1 /(1-\alpha) r \in\left[\mu^{m}, \mu^{M}\right)$.

Proof. As we proved in Proposition 3.1, part (b), if $\mu \in\left[\mu^{m}, \mu^{M}\right)$, then the unique fixed point $W_{2}^{\star}$ belongs to $D_{2}$ so its multiplier is given by (3.3) that belongs to $(-1,0) \Leftrightarrow \mu<\mu^{\star}$. It is straightforward to conclude that the fixed point is also globally stable.

Now we have to study the case of $\mu \in\left[\mu^{\star}, \mu^{M}\right)$. A previous consideration is that if $\mu=\mu^{\star}$, then the unique fixed point $W_{2}^{\star}$ is not hyperbolic: its multiplier, given by (3.3) is in fact $\left.f_{2}^{\prime}\left(W_{2}^{\star}\right)\right|_{\mu=\mu^{\star}}=-1$. So, when $\mu=\mu^{\star}$, the map exhibits a bifurcation: its fixed point becomes unstable and we have to identify the new attractor that is eventually born.

Before studying this case, we consider that the map $f$ is piecewise linear so it is only piecewise smooth and it can exhibit noncanonical bifurcation phenomena. While in the wellknown period-doubling route to chaos when a fixed point becomes unstable, we observe a period-two stable orbit; in such a case this does not happen even if we find out a cycle-2 owned by the map as proved in the following proposition.

Proposition 3.4. Let $f$ be given by (2.14) and let $e>0$ and $(1-\alpha) r<1$. Let also $\mu=\mu^{\star}$. Then there exists a period-2 orbit, say $\mathrm{O}_{2}$, that involves the maximum point, given by (2.12), that is, $\mathrm{O}_{2}=$ $\left\{W^{M}, f\left(W^{M}\right)\right\}$.

Proof. The proof is straightforward as it is only based on the computation that $\left.f^{2}\left(W^{M}\right)\right|_{\mu=\mu^{\star}}=$ $\left.W^{M}\right|_{\mu=\mu^{\star}}$. 


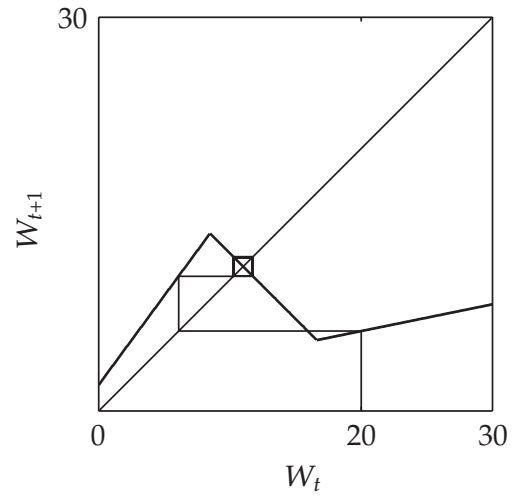

(a)

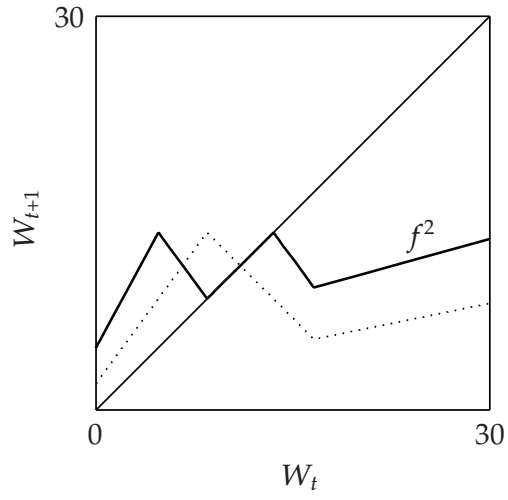

(b)

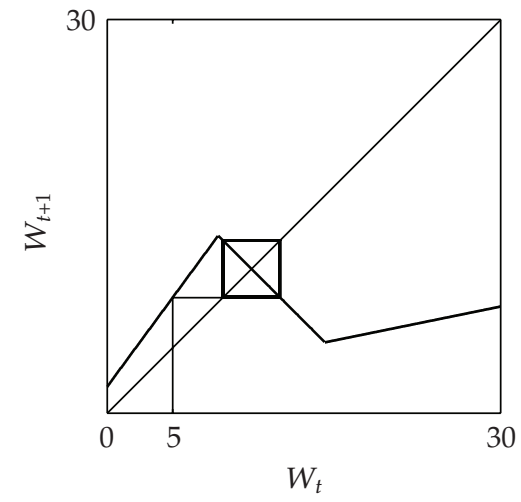

(c)

Figure 4: Repelling period-2 orbits for $\mu \simeq 4.9$. In (a), we have a first cycle two for the initial condition $W_{0}=20$ that is different from the one in (c) for $W_{0}=5$. In (b), the fixed invariant interval of $f^{2}$ is illustrated.

Once known that $f$ has a cycle-2 involving the maximum point for the bifurcation value $\mu=\mu^{\star}$, we are interested in knowing if such invariant set is stable. However, since the map is not differentiable in $W^{M}$, we cannot compute its multiplier so we cannot study its stability in the typical way.

The discontinuity in the first derivative of the map implies that it can jump without crossing the bifurcation value -1 , so an attractor could die without a double-period one being born. Furthermore, as proved in Nusse and Yorke [20], border collision bifurcations are possible so that the map could pass from a stable fixed point to a variety of attractors like a period- $m$ attractor $(m \geq 2)$, or a $2 m$-piece chaotic attractor, or a $m$-piece chaotic attractor or finally a one-piece chaotic attractor.

In order to study the stability of the cycle-2, $O_{2}$, we found out for $\mu=\mu^{\star}$, we have to consider that the point $W^{M}$ that belongs to $\mathrm{O}_{2}$ has no derivative (it has two one-sided derivatives). However, the following proposition proves that the bifurcation parameter value $\mu^{\star}$ opens a chaotic region via a border collision bifurcation.

Proposition 3.5. Let $f$ be given by (2.14) and let $e>0$ and $(1-\alpha) r<1$. Let also $\mu=\mu^{\star}$. Then $W^{M}$ is a Misiurewicz point. (A preperiodic point is usually called a Misiurewicz point.) 


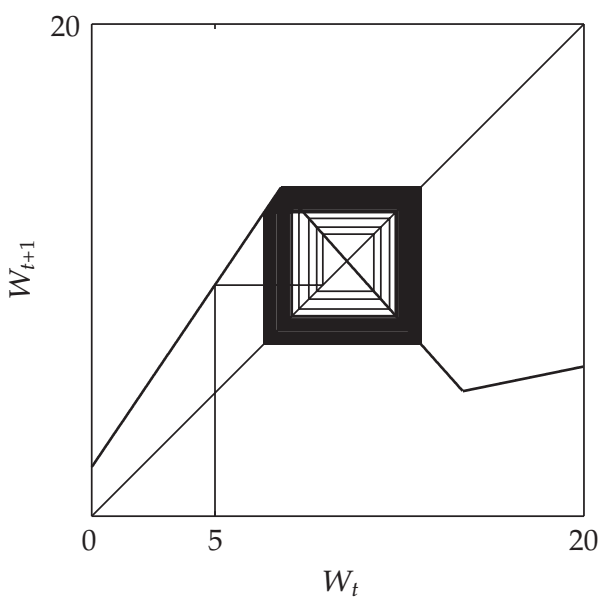

(a)

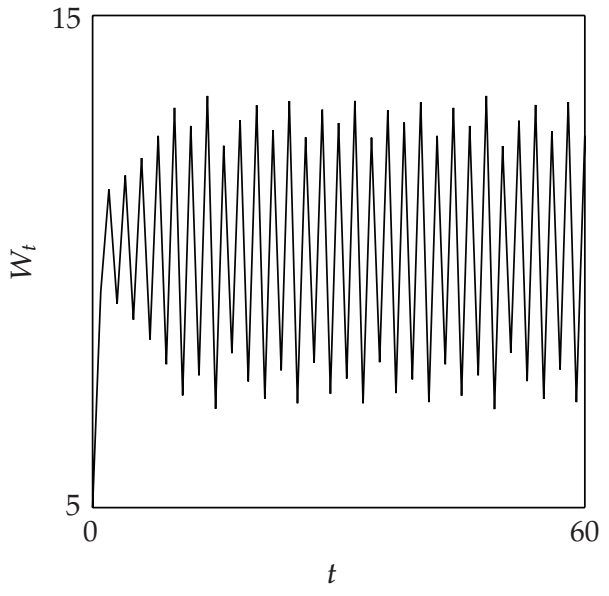

(b)

Figure 5: (a) The generic aperiodic orbit covers two disjoint invariant sets. (b) The trajectory with respect to time. In both cases $\mu=5.5$ and $W_{0}=5$.

Proof. For all $W_{t} \in\left[W^{M}, f\left(W^{M}\right)\right]$, we have that $f^{2}\left(W_{t}\right)=W_{t}$ (it can be verified by simple calculations, that is, $\left.f^{2}\left(W_{t}\right)=\left.f_{2} \circ f_{2}\right|_{\mu=\mu^{\star}}=W_{t}\right)$ as a consequence of the fact that $f^{\prime}\left(W_{2}^{\star}\right)=-1$. So each point in the set $I_{2}=\left[W^{M}, f\left(W^{M}\right)\right]$ is a fixed point for the second iterate, $f^{2}$. Then each point in $I_{2}$, except $W_{2}^{\star}$, is involved by a cycle- 2 and each of such periodic orbits must be unstable, so also $\mathrm{O}_{2}$ is an unstable period-two orbit. Because $\mathrm{O}_{2}$ involves the maximum $W^{M}$, as we proved in Proposition 3.4, then the critical point is attracted by an unstable orbit so it is a Misiurewicz point.

Since the topological entropy at the Misiurewicz point is greater than 1, it reveals that we have entered into a (aperiodic) chaotic region. (At the preperiodic point, we have no attracting cycles since they cannot capture the critical point, which is preperiodic.) In particular, after the bifurcation occurred at $\mu^{\star}$, the map is 2-piece chaotic.

Here we cannot prove other results with respect to all the parameters of the system however, since other qualitative dynamics that could eventually emerge strictly depend on the fixed values of the parameters, in Section 4 we use the numerical analysis to support the results we derived in this section and we present numerical simulations fixing all the parameters but $\mu$ at economically plausible values. In such a way, we pursue numerical results about the dynamics exhibited by the model. The quantitative analysis allow us to show the dynamic evolution of the system and to conclude about its properties.

\section{Quantitative dynamics}

In this section, we provide some numerical simulations by fixing the values of all the parameters of the model but $\mu$. In such a way, we are able to prove quantitatively the qualitative results reached in Section 3 and also to pursue other results that cannot be proved rigorously. Let $\alpha=0.8, r=1.02, a=0.5$ so $1 / a=2, e=10$, and $Z=100$. (As it can be proved, the chosen value of $Z$ only affects the quantitative dynamics, that is, the width of the invariant interval where the dynamics are exhibited, but not the qualitative dynamics, 


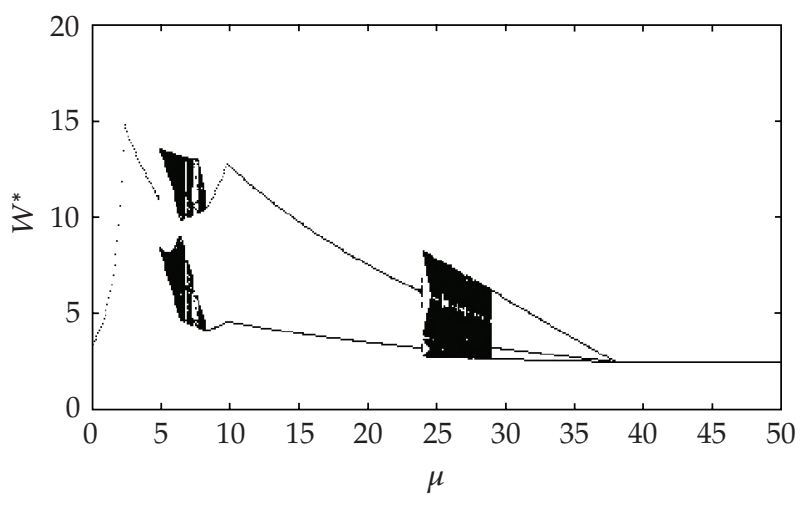

Figure 6: Bifurcation diagram with respect to $\mu$.

that is, the bifurcation sequence occurs at the same parameter values of $\mu$. We choose these parameter values according to what is considered in Aghion et al. [3].)

In Figure 1, we present the scheme of $f$ for different values of $\mu$. As we proved in Proposition 3.1, the fixed point can belong to each of the pieces of $f$ depending on $\mu$, where $\mu^{m} \simeq 2.37$ while $\mu^{M} \simeq 39.02$. (We are approximating an error less than $10^{-2}$.)

As we proved in Proposition 3.2 the economy is globally stable for $0 \leq \mu<\mu^{m}$ and $\mu \geq$ $\mu^{M}$ and the generic orbit definitively converges monotonically to $W_{1}^{\star}$ or $W_{3}^{\star}$, as determined in (3.4) and (3.6), that are points in which $f$ is increasing. In fact, the Koenigs Lemerary staircase diagram in Figures 2 and 3 shows the converging trajectory for an arbitrary initial condition.

As we proved in Proposition 3.1, the fixed point $W_{2}^{\star}$ belongs to the decreasing piece when $\mu^{m} \leq \mu<\mu^{M}$ that is the case of economies going through a phase of financial development. Furthermore, because of the bimodality of $f$, there is a stretching and folding action that could generate complex dynamics like cycles of every period and chaos. However, as we stated in Proposition 3.3, if $\mu<\mu^{\star}$, where $\mu^{\star} \simeq 4.9$, the fixed point is still globally stable even if the generic orbit is asymptotically oscillating.

In case $\mu=\mu^{\star}$ the map exhibits a bifurcation and it gives rise to an infinite number of repelling period-2 cycles. In fact, Propositions 3.4 and 3.5 show such evidence. Note that for such value of $\mu$, the fixed point is not hyperbolic while each point $W_{t} \in\left[W^{M}, f\left(W^{M}\right)\right]$ is fixed for the second iterate of $f$, as it is clear when looking at Figure 4(b). So all the period-2 orbits are unstable. Furthermore, the set $\left[W^{M}, f\left(W^{M}\right)\right]$ is positively invariant, so every initial condition will converge to one of such repelling periodic orbit.

Numerical computations also show that all these cycles-2 are of the kind $O_{2}=\left\{W_{2}^{\star}+\right.$ $\left.\gamma, W_{2}^{\star}-\gamma\right\}$, for all $\gamma \in\left(0,\left(f\left(W^{M}\right)-W^{M}\right) / 2\right]$, where $W_{2}^{\star}$ is given by (3.5). Two of such orbits are, for example, the ones in Figure 4(a) and 4(c). The bifurcation occurring at $\mu^{\star}$ is not canonical: one of the repelling cycle-2 involves the maximum point $W^{M}$ that is a Misiurewicz point. Therefore, such border-collision bifurcation opens a chaotic region in which the generic orbit covers two disjoint invariant sets. Figure 5 shows the trajectory for an initial condition once the bifurcation happens; the trajectory is also represented versus time.

As we said, after the bifurcation at $\mu^{\star}$, the dynamics are suddenly chaotic so the map has the properties of density of the periodic orbits, topological transitivity, and sensitively dependence on the initial condition. (We are referring to the definition of chaotic set given in Devaney [26].) 


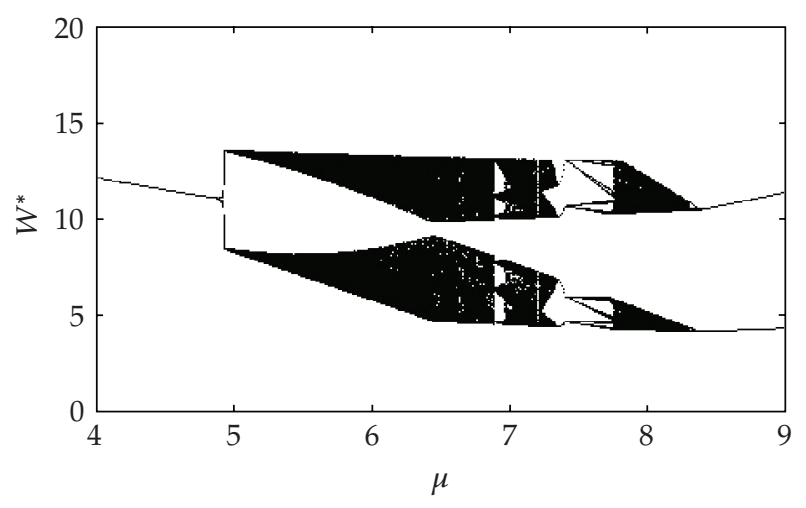

Figure 7: Bifurcation diagram for $\mu \in(4,9)$.

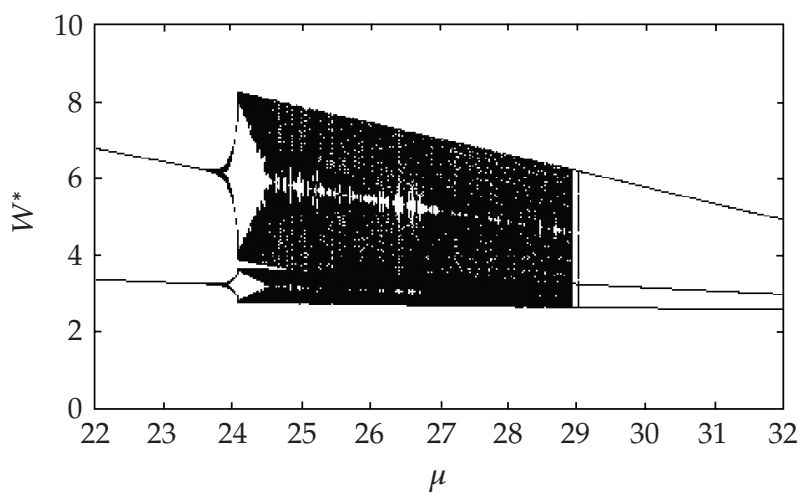

Figure 8: Bifurcation diagram for $\mu \in(22,32)$.

Figure 6 shows the bifurcation diagram of the map for different values of $\mu$. The black intervals are those in which the dynamics is chaotic or periodic with very high period. Furthermore, we observe both large intervals of $\mu$ where the asymptotic behavior is a cycle-2 (if $\mu \in\left(\mu_{1}, \mu_{2}\right)$ with $\mu_{1} \simeq 8.39$ and $\mu_{2} \simeq 23.5$ ) or a cycle-3 (if $\mu \in\left(\mu_{1}^{\prime}, \mu_{2}^{\prime}\right)$ with $\mu_{1}^{\prime} \simeq 29.1$ and $\mu_{2}^{\prime} \simeq 39.02$ ). (In such a case, the chaotic properties could also be proven by the well-known $\mathrm{Li}$ and Yorke Theorem, see Li and Yorke [27].) In this case, the dynamics are still predictable even in the long run.

However, inside the two chaotic regions, that are visible in the following Figures 7 and 8 , the dynamics are both chaotic and periodic with eventually a very high period. So, while in the first case the asymptotic behavior is not predictable, in the second case it is still predictable even though the attractor could be nonhyperbolic, and so the system would be structurally unstable. Some small intervals of $\mu$ that are periodic windows inside the chaotic region are visible in such figures.

\section{Conclusions}

In this work, we studied a piecewise linear dynamic system describing a small open economy where the reached level of financial development plays a central role as a source of endogenous instability. 
By analyzing the qualitative dynamics, we proved rigorously the global stability of economies at a low or high level of financial development. On the contrary, the economies at an intermediate level of financial development could not converge to the steady state. Consequently, we assess the existence of chaotic behavior in the patterns. In this case, we have been able to prove by qualitative and also quantitative study the following results.

(i) Economies at an intermediate level of financial development eventually converge to the fixed point by oscillations or they fluctuate indefinitely.

(ii) They can be unstable but predictable if the attractor is a stable periodic orbit, even with high period, that can also belong to a window in the chaotic region.

(iii) They can be unstable and unpredictable if we are in a proper chaotic region because of the sensitivity to the initial conditions.

(iv) Economies can be structurally unstable when going trough regions governed by different asymptotic dynamics because of the lack of hyperbolicity.

(v) The bifurcation phenomenon is atypical because of the presence of no differentiable points.

The instability of economies that are financially developing can be understood according to the hypothesis of the model studied. In fact, during a boom, the investment expands and so does the demand for the country-specific factor. It increases its price and pushes down future profits. Less profits lead to less creditworthiness because of the presence of the credit constraint and consequently less investments. Finally, the country-specific factor will not be completely exhausted so its prices will fall down with high future profits and a new possible economic boom.

\section{References}

[1] World Economic Outlook, Financial Crises: Characteristic and Indicators of Vulnerability, chapter 4, International Monetary Fund, Washington, DC, USA, 1998.

[2] G. Corsetti, P. Pesenti, and N. Roubini, "What caused the Asian currency and financial crisis?" Japan and the World Economy, vol. 11, no. 3, pp. 305-373, 1999.

[3] P. Aghion, P. Bacchetta, and A. Banerjee, "Capital markets and the instability of open economies," Discussion paper 2083, CEPR, London, UK, 1999.

[4] P. Aghion, P. Bacchetta, and A. Banerjee, "A simple model of monetary policy and currency crises," European Economic Review, vol. 44, no. 4-6, pp. 728-738, 2000.

[5] P. Aghion, P. Bacchetta, and A. Banerjee, "A corporate balance-sheet approach to currency crises," Discussion paper 3092, CEPR, London, UK, 2001.

[6] R. J. Caballero and A. Krishnamurthy, "A simple model of monetary policy and currency crises," Working paper 7971, NBER, Cambridge, Mass, USA, 2000.

[7] L. F. Cespedes, R. Chang, and A. Velasco, "Balance sheets and the exhange rate policy," Working paper 7840, NBER, Cambridge, Mass, USA, 2000.

[8] P. Krugman, "Balance sheet, the transfer problem and financial crises," in International Finance and Financial Crises: Essay in Honor of R. P. Flood, P. Isard, A. Razin, and A. Rose, Eds., Kluwer Academic Publishers, Dordrecht, The Netherlands, 1999.

[9] B. Bernanke and M. Gertler, "Agency costs, net worth, and business fluctuations," American Economic Review, vol. 79, no. 1, pp. 14-31, 1989.

[10] J. Furman and J. E. Stiglitz, "Economic crises: evidence and insights from East Asia," Brookings Papers on Economic Activity, no. 2, pp. 1-135, 1998.

[11] F. S. Mishkin, "Global financial instability: framework, events, issues," Journal of Economic Perspectives, vol. 13, no. 4, pp. 3-20, 1999.

[12] R. G. Hubbard, "Capital-market imperfections and investment," Journal of Economic Literature, vol. 36, no. 1, pp. 193-225, 1998. 
[13] B. Bernanke, M. Gertler, and S. Gilchirist, "The financial accelerator in a quantitative business cycle framework," in Handbook of Macroeconomics, J. B. Taylor and M. Woodford, Eds., pp. 1341-1393, Elsevier, Amsterdam, The Netherlands, 1998.

[14] C. Azariadis and B. Smith, "Financial intermediation and regime switching in business cycles," American Economic Review, vol. 88, no. 3, pp. 516-536, 1998.

[15] N. Kiyotaki and J. Moore, "Credit cycles," Journal of Political Economy, vol. 105, no. 2, pp. 211-248, 1997.

[16] P. Aghion, P. Bacchetta, and A. Banerjee, "Currency crises and monetary policy in an economy with credit constraints," European Economic Review, vol. 45, no. 7, pp. 1121-1150, 2001.

[17] P. Aghion, A. Banerjee, and T. Piketty, "Dualism and macroeconomic volatility," Quarterly Journal of Economics, vol. 114, no. 4, pp. 1359-1397, 1999.

[18] P. Aghion, P. Bacchetta, and A. Banerjee, "Financial development and the instability of open economies," Journal of Monetary Economics, vol. 51, no. 6, pp. 1077-1106, 2004.

[19] J. Caballé, X. Jarque, and E. Michetti, "Chaotic dynamics in credit constrained emerging economies," Journal of Economic Dynamics E Control, vol. 30, no. 8, pp. 1261-1275, 2006.

[20] H. E. Nusse and J. A. Yorke, "Border-collision bifurcations for piecewise smooth one-dimensional maps," International Journal of Bifurcation and Chaos, vol. 5, no. 1, pp. 189-207, 1995.

[21] J. Galvez and J. Tybout, "Microeconomic adjustments in Chile during 1977-1981: the importance of being a grupo," World Development, vol. 13, no. 8, pp. 969-994, 1985.

[22] A. H. Petrei and J. Tybout, "Microeconomic adjustments in Argentina during 1976-1981: the importance of changing levels of financial subsidies," World Development, vol. 13, no. 8, pp. 949-967, 1985.

[23] J. De Melo, R. Pascale, and J. Tybout, "Microeconomic adjustments in Uruguay during 1973-1981: the interplay of real and financial shocks," World Development, vol. 13, no. 8, pp. 995-1015, 1985.

[24] The World Bank, Private Capital Flows to Developing Countries, World Bank Policy Report, Oxford University Press, Oxford, UK, 1997.

[25] B. Holmstrom and J. Tirole, "Financial intermediation, loanable funds, and the real sector," Quarterly Journal of Economics, vol. 112, no. 3, pp. 663-691, 1997.

[26] R. L. Devaney, An Introduction to Chaotic Dynamical Systems, The Benjamin/Cummings, Menlo Park, Calif, USA, 1986

[27] T.-Y. Li and J. A. Yorke, "Period three implies chaos," The American Mathematical Monthly, vol. 82, no. 10, pp. 985-992, 1975. 


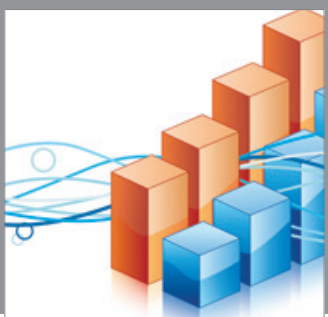

Advances in

Operations Research

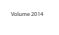

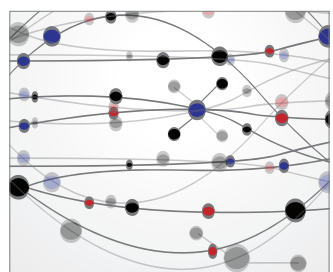

\section{The Scientific} World Journal
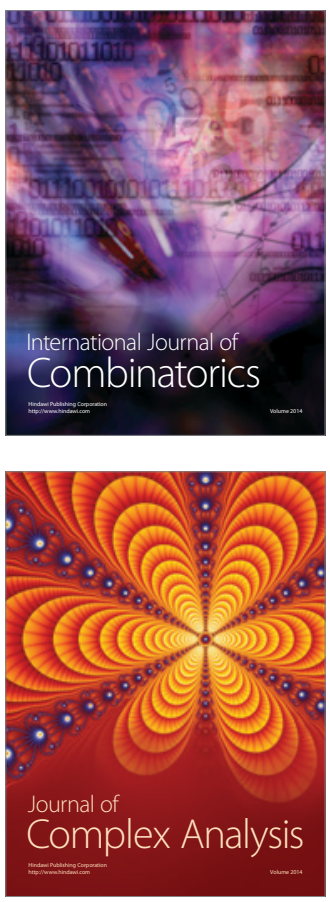

International Journal of

Mathematics and

Mathematical

Sciences
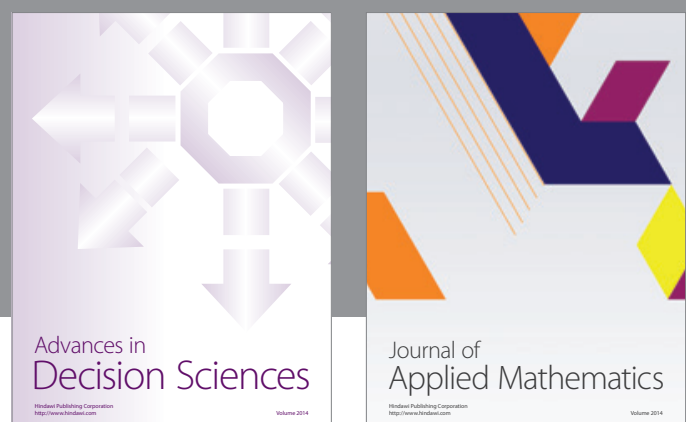

Journal of

Applied Mathematics
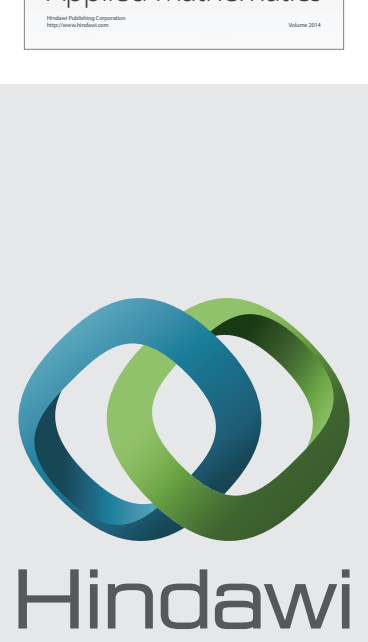

Submit your manuscripts at http://www.hindawi.com
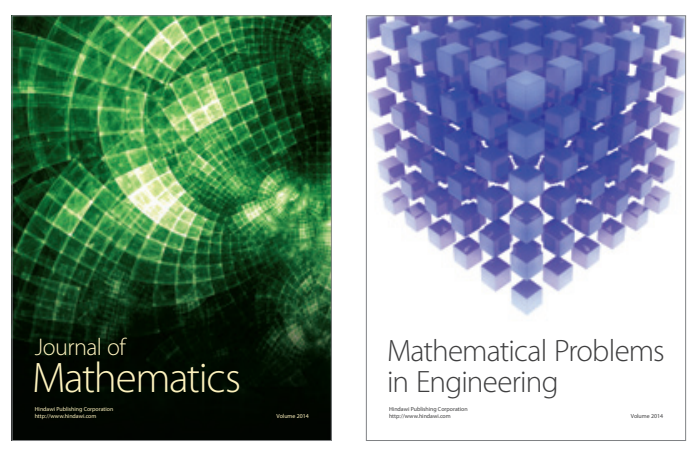

Mathematical Problems in Engineering
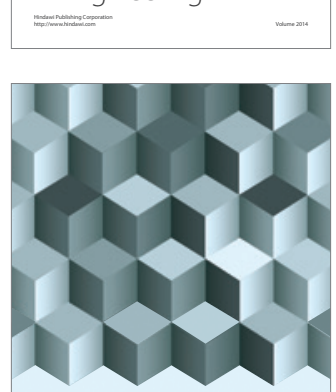

Journal of

Function Spaces
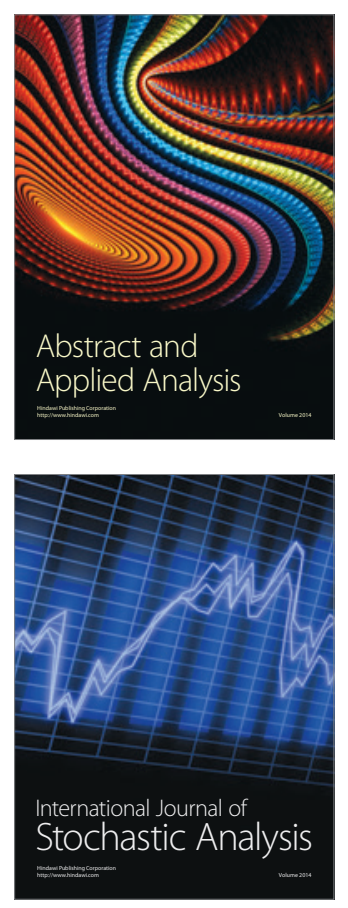

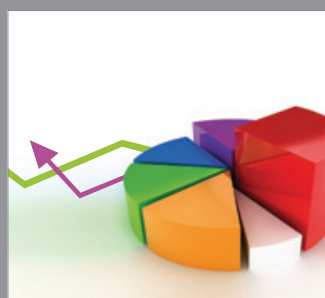

ournal of

Probability and Statistics

Promensencen
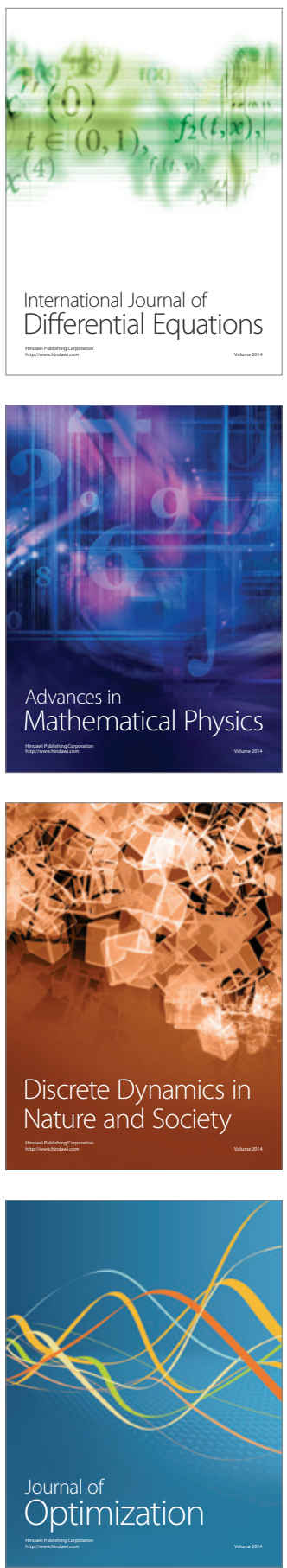